Reprod. Nutr. Dévelop., 1982, 22 (1 A), 123-134.

\title{
Importance du noyau ventro-médian de l'hypothalamus dans la régulation du comportement alimentaire de l'oie de race landaise
}

\author{
J.-C MARCILLOUX, P. AUFFRAY
}

avec la collaboration technique de L. ROYER

Laboratoire de Physiologie de la Nutrition, I.N.R.A., 78350 Jouy-en-Josas, France.

Summary. Role of the ventromedial nucleus of the hypothalamus in regulating feeding behaviour in Landes geese.

This study shows the apparent role of the ventromedial nucleus of the hypothalamus (VMH) in regulating food intake in the goose. The feeding pattern of normal animals has been compared with that of animals made hyperphagic by destruction of the $\mathrm{VMH}$. Lesioning of the VMH modified the circadian pattern of intake. All the geese tended to equalize their intakes between the light and dark periods. The overall effect of lesioning was considerable hyperphagia resulting from an increase in the number of meals and of their amplitude and duration, as well as in the rate of ingestion. We have emphasized the importance of defining the meal when interpreting changes in the correlations between the amount of food ingested during the meal and the length of pre or postprandial intervals after destruction of the $\mathrm{VMH}$.

Although it is important, the fatty liver syndrome induced after hyperphagia is not sufficient to produce fatty livers which can compete with those obtained by traditional cramming.

\section{Introduction.}

Depuis une trentaine d'années, de nombreuses études, entreprises essentiellement chez les mammifères, rapportent l'existence d'une hyperphagie consécutive à la lésion des noyaux ventro-médians de l'hypothalamus. Chez les oiseaux, la surconsommation engendrée par la destruction de cette structure n'a pas toujours été évidente. C'est ainsi que Lepkovsky et Yasuda (1966), Lepkovsky et al. (1967) chez le Poulet ainsi que Hawkes et George (1975) chez le Canard obtiennent plutôt de l'obésité que de l'hyperphagie. Un accroissement de la prise alimentaire est cependant enregistré par Kuenzel et Helms (1967) chez le Moineau ainsi que par Snapir et al. (1973) chez le Poulet, mais il semble que ce soit chez l'Oie que l'on ait induit pour la première fois une hyperphagie marquée accompagnée d'une stéatose hépatique (Auffray et Blum, 1970 ; Auffray et al., 1973 ; Snapir et al., 
1976). Le déroulement de cette hyperphagie provoquée n'est pas sans rappeler celui obtenu chez le Rat, la phase dynamique étant cependant nettement plus marquée en amplitude chez l'Oie, mais de durée plus courte (12 à 15 jours). II s'ensuit une surconsommation globale trop faible pour l'obtention d'un foie gras répondaṇt aux normes traditionnelles. Aucun travail ne relate les particularités du comportement alimentaire de cet animal durant cette période. II est donc intéressant, sur un plan théorique, après avoir enregistré le profil alimentaire de l'Oie normale (Marcilloux et Auffray, 1981) de déterminer celui-ci au cours de la phase dynamique d'hyperphagie. A la lumière de ces données nouvelles, ił serait peutêtre possible d'intervenir sur l'obésité et par voie de conséquence sur le développement du foie.

\section{Matériel et méthodes.}

17 Oies de race Landaise, adultes, de 5 à 6 mois d'âge et de poids moyen de $5,4 \pm 0,2 \mathrm{~kg}$ sont utilisées au cours de cette expérimentation.

Techniques chirurgicale et histologique. - Les noyaux ventro-médians de I'hypothalamus (VMH) sont détruits par coagulation électrolytique anodique après repérage stéréotaxique, selon la méthode décrite par Auffray et Blum (1970). Les animaux sont abattus à la fin de la phase d'hyperphagie dynamique, des coupes sériées sont effectuées à congélation, sur les cerveaux des animaux lésés, puis colorées suivant la technique de Nissl (Chevreau et al., 1977).

\section{Protocole expérimental.}

- Un lot de 13 animaux porteurs de lésions médio-ventrales hypothalamiques est étudié.

- Un lot de 4 animaux soumis à la même intervention, mais non porteurs de lésion, est étudié dans les mêmes conditions constituant ainsi le lot témoin.

Comme nous l'avions noté antérieurement les Oies sont divisées en deux groupes en fonction de la partie du nycthémère où l'ingestion est la plus élevée. Le lot des animaux ayant subi la lésion est composé de 9 individus à tendance diurne et de 4 individus à tendance nocturne, le lot témoin étant composé de 3 individus à tendance diurne et de 1 à tendance nocturne. Ĺexpérimentation consiste à comparer le profil alimentaire de l'Oie normale avec celui de l'Oie rendue hyperphagique. Le comportement alimentaire de ces animaux est analysé péndant une période de 13 jours avant l'intervention ; cet enregistrement met en évidence les tendances diurne et nocturne de chacun d'eux. Ces mêmes animaux sont ensuite observés après production de la lésion durant une période de 13 jours qui correspond à la durée de la phase dynamique d'hyperphagie. L'enregistrement des consommations alimentaires recommence dès le lendemain de l'intervention chirurgicale.

La technique d'enregistrement des prises alimentaires ainsi que les conditions d'alimentation sont analogues à celles décrites antérieurement (Marcilloux et Auffray, 1981). Rappelons que l'aliment est distribué sous forme semi-liquide et que 
le cycle lumineux 12/12 est maintenu pendant toute la durée de l'expérimentation. Par convention, deux prises alimentaires espacées de moins de $7 \mathrm{~min}$. $30 \mathrm{~s}$ sont groupées en un seul repas.

Les résultats recueillis sont comparés par l'analyse de variance et les histogrammes par le test 2 î Kupperman (1959).

\section{Résultats expérimentaux.}

Etude des modifications du profil alimentaire apportées par la lésion.

1. Modifications de la répartition temporelle de la consommation et valeur des divers paramètres après la lésion.

- Chez les Oies lésées, nous observons une augmentation moyenne de 144 p. 100 des quantités journalières consommées, ceci entraînant un gain de poids total de 1,140 $\pm 0,13 \mathrm{~kg}$ de ces animaux.

- Chez les Oies témoins, l'augmentation de l'ingéré n'est que de 8 p. 100 avec un gain de poids total de $0,070 \pm 0,050 \mathrm{~kg}$.

\section{TABLEAU 1}

Valeurs des accroissements des quantités d'aliment consommées et du nombre de repas quotidiens après la lésion des noyaux VMH

\begin{tabular}{|c|c|c|c|c|c|c|c|}
\hline \multirow[b]{2}{*}{$\begin{array}{c}\text { Groupes } \\
\text { d'animaux }\end{array}$} & \multirow[b]{2}{*}{$\begin{array}{l}\text { Période du } \\
\text { nycthémère }\end{array}$} & \multicolumn{3}{|c|}{ Quantités d'aliment consommées } & \multicolumn{3}{|c|}{ Nombre de repas } \\
\hline & & $\begin{array}{l}\text { Avant } \\
\text { lésion }\end{array}$ & $\begin{array}{l}\text { Après } \\
\text { lésion }\end{array}$ & $\begin{array}{c}\text { p. } 100 \\
\text { d'augmentation }\end{array}$ & $\begin{array}{l}\text { Avant } \\
\text { lésion }\end{array}$ & $\begin{array}{l}\text { Après } \\
\text { lésion }\end{array}$ & $\begin{array}{c}\text { p. } 100 \\
\text { d'augmentation }\end{array}$ \\
\hline $\begin{array}{l}\text { Tendance } \\
\text { diurne }(9)\end{array}$ & $\begin{array}{l}\text { Lumière } \\
\text { obscurité }\end{array}$ & $\begin{array}{r}119 \mathrm{~g} \\
76 \mathrm{~g}\end{array}$ & $\begin{array}{l}252 \mathrm{~g} \\
249 \mathrm{~g}\end{array}$ & $\begin{array}{l}112 \\
227\end{array}$ & $\begin{array}{r}10,2 \text { repas } \\
6,4 \text { repas }\end{array}$ & $\begin{array}{l}12,2 \text { repas } \\
12,2 \text { repas }\end{array}$ & $\begin{array}{l}20 \\
91\end{array}$ \\
\hline $\begin{array}{c}\text { Tendance } \\
\text { nocturne }\{4\}\end{array}$ & $\begin{array}{l}\text { Lumière } \\
\text { obscurité }\end{array}$ & $\begin{array}{r}93 \mathrm{~g} \\
128 \mathrm{~g}\end{array}$ & $\begin{array}{l}275 \mathrm{~g} \\
251 \mathrm{~g}\end{array}$ & $\begin{array}{r}196 \\
96\end{array}$ & $\begin{array}{l}7,3 \text { repas } \\
9,9 \text { repas }\end{array}$ & $\begin{array}{l}14,0 \text { repas } \\
15,6 \text { repas }\end{array}$ & $\begin{array}{l}92 \\
58\end{array}$ \\
\hline
\end{tabular}

L'accroissement de consommation se produit au cours des deux phases du cycle nycthéméral (tabl. 1) ; cet accroissement est cependant plus marqué au cours de la période pendant laquelle l'animal consommait le moins avant la lésion. La figure 1 met en évidence ces modifications et permet de constater la rupture du cycle nychtéméral avec une tendance à l'égalisation des consommations journuit. Les pics de consommation, caractéristiques chez I'animal normal, n'apparaissent plus chez l'animal après lésion du VMH. Le tableau 2 qui folirnit les valeurs de tous les paramètres étudiés montre que l'accroissement des quantités journalières consommées est le résultat de l'augmentation à la fois du nombre de repas, de leur durée, de leur amplitude et de la vitesse à laquelle ils se déroulent. L'analyse de variance effectuée sur les résultats obtenus nous permet de dire que le traitement provoque une augmentation significative des valeurs de tous les paramètres étudiés (mis à part la durée de l'intervalle entre deux repas qui a diminué) et que dans l'ensemble il n'y a pas de variation individuelle dans la réponse 
Moyennes et écarts types de la moyenne des divers paramètres caractérisant le comportement alimentaire de l'Oie

Animaux avec lésions $\mathrm{VMH}$

Paramètres étudiés

$\begin{array}{cc}\text { Avant } & \text { Après } \\ \text { lésions } & \text { lésions }\end{array}$

Analyse de variance

lésions lésions

Effet Effet

Animal traitement

\begin{tabular}{|c|c|c|c|c|c|c|c|}
\hline \multirow{3}{*}{$\begin{array}{l}\text { Ingéré en } \\
\mathrm{g} \text { de farine }\end{array}$} & $\begin{array}{l}\text { A Sur l'ensemble du nycthémère } \\
\text { tous les animaux groupés (a) }\end{array}$ & 207 & \pm 15 & 505 & \pm 36 & N.S. & *** \\
\hline & $\begin{array}{l}\text { B Partie éclairée } \\
\text { 1) Chez l'animal à tendance diurne } \\
\text { 2) Chez l'animal à tendance nocturne }\end{array}$ & $\begin{array}{r}119 \\
93\end{array}$ & $\begin{array}{l} \pm 13 \\
\pm 10\end{array}$ & $\begin{array}{l}252 \\
275\end{array}$ & $\begin{array}{l} \pm 26 \\
\pm 27\end{array}$ & $\begin{array}{l}\text { N.S. } \\
\text { N.S. }\end{array}$ & ** \\
\hline & $\begin{array}{l}\text { C Partie obscure } \\
\text { 1) Chez l'animal à tentance diurne } \\
\text { 2) Chez l'animal à tendance nocturne }\end{array}$ & $\begin{array}{r}76 \\
128\end{array}$ & $\begin{array}{l} \pm 8 \\
\pm 11\end{array}$ & $\begin{array}{l}249 \\
251\end{array}$ & $\begin{array}{l} \pm 25 \\
\pm 17\end{array}$ & $\begin{array}{l}\text { N.S. } \\
\text { N.S. }\end{array}$ & ** \\
\hline \multirow{3}{*}{$\begin{array}{l}\text { Nombre } \\
\text { de repas }\end{array}$} & $\begin{array}{l}\text { A Sur l'ensemble du nycthémère } \\
\text { tous les animaux groupés (a) }\end{array}$ & 16,9 & $\pm 1,5$ & 26,3 & $\pm 2,0$ & N.S. & *** \\
\hline & $\begin{array}{l}\text { B Partie éclairée } \\
\text { 1) Chez l'animal à tendance diurne } \\
\text { 2) Chez l'animal à tendance nocturne }\end{array}$ & $\begin{array}{r}10,2 \\
7,3\end{array}$ & $\begin{array}{l} \pm 1,1 \\
\pm \quad 1,7\end{array}$ & $\begin{array}{l}12,2 \\
14,0\end{array}$ & $\begin{array}{l} \pm \quad 1,6 \\
\pm \quad 1,0\end{array}$ & N.S. & $\underset{*}{\text { N.S. }}$ \\
\hline & $\begin{array}{l}\text { C Partie obscure } \\
\text { 1) Chez l'animal à tendance diurne } \\
\text { 2) Chez l'animal à tendance nocturne }\end{array}$ & $\begin{array}{l}6,4 \\
9,9\end{array}$ & $\begin{array}{l} \pm 0,8 \\
\pm \quad 1,5\end{array}$ & $\begin{array}{l}12,2 \\
15,6\end{array}$ & $\begin{array}{l} \pm \quad 0,9 \\
\pm \quad 1,9\end{array}$ & N.S. & N.S. \\
\hline \multirow{3}{*}{$\begin{array}{l}\text { Durée d'un } \\
\text { repas (en s) }\end{array}$} & $\begin{array}{l}\text { A Sur l'ensemble du nycthémère } \\
\text { tous les animaux groupés (a) }\end{array}$ & 123 & \pm 2 & 172 & \pm 2 & * & * \\
\hline & $\begin{array}{l}\text { B Partie éclairée } \\
\text { 1) Chez l'animal à tendance diurne } \\
\text { 2) Chez l'animal à tendance nocturne }\end{array}$ & $\begin{array}{l}111 \\
185\end{array}$ & $\begin{array}{l} \pm 18 \\
\pm 30\end{array}$ & $\begin{array}{l}149 \\
208\end{array}$ & $\begin{array}{l} \pm 30 \\
\pm 22\end{array}$ & N.S. & $\begin{array}{l}\text { N.S. } \\
\text { N.S. }\end{array}$ \\
\hline & $\begin{array}{l}\text { C Partie obscure } \\
\text { 1) Chez l'animal à tendance diurne } \\
\text { 2) Chez l'animal à tendance nocturne }\end{array}$ & $\begin{array}{l}120 \\
175\end{array}$ & $\begin{array}{l} \pm 19 \\
\pm 40\end{array}$ & $\begin{array}{l}174 \\
192\end{array}$ & $\begin{array}{l} \pm 29 \\
\pm 18\end{array}$ & N.S. & N.S. \\
\hline \multirow{3}{*}{$\begin{array}{l}\text { Durée d'un } \\
\text { intervalle } \\
\text { (en min.) }\end{array}$} & $\begin{array}{l}\text { A Sur l'ensemble du nycthémère } \\
\text { Tous les animaux groupés (a) }\end{array}$ & 74 & \pm 1 & 48 & $\pm 0,7$ & * & *** \\
\hline & $\begin{array}{l}\text { B Partie éclairée } \\
\text { 1) Chez l'animal à tendance diurne } \\
\text { 2) Chez l'animal à tendance nocturne }\end{array}$ & $\begin{array}{l}71 \\
91\end{array}$ & $\begin{array}{l} \pm 9 \\
\pm 19\end{array}$ & $\begin{array}{l}58 \\
47\end{array}$ & $\begin{array}{l} \pm 12 \\
\pm \quad 6\end{array}$ & N.S. & $\begin{array}{l}\text { N.S. } \\
\text { N.S. }\end{array}$ \\
\hline & $\begin{array}{l}\text { C Partie obscure } \\
\text { 1) Chez l'animal à tendance diurne } \\
\text { 2) Chez l'animal à tendance nocturne }\end{array}$ & $\begin{array}{r}102 \\
61\end{array}$ & $\begin{array}{l} \pm 11 \\
\pm 11\end{array}$ & $\begin{array}{l}54 \\
42\end{array}$ & $\begin{array}{l} \pm 4 \\
\pm 8\end{array}$ & $\begin{array}{l}\text { N.S. } \\
\text { N.S. }\end{array}$ & N.S. \\
\hline \multirow{3}{*}{$\begin{array}{l}\text { Ingéré } \\
\text { par repas } \\
\text { (en g) }\end{array}$} & Sur l'ensemble du nycthémère (b) & 10,2 & $\pm 0,3$ & 20,6 & $\pm \quad 0,4$ & & \\
\hline & Sur la partie éclairée & 9,9 & $\pm \quad 0,4$ & 23,6 & $\pm \quad 0,6$ & & \\
\hline & Sur la partie obscure & 10,8 & $\pm 0,6$ & 17,8 & $\pm 0,5$ & & \\
\hline \multirow{3}{*}{$\begin{array}{l}\text { Vitesse } \\
\text { d'ingestion }^{\prime} \\
\text { (en } \mathrm{g} / \mathrm{min} . \text { ) }\end{array}$} & Sur l'ensemble du nycthémère (b) & 9,7 & $\pm \quad 0,9$ & 9,9 & $\pm 0,5$ & & \\
\hline & Sur la partie éclairée & 11,6 & $\pm 1,3$ & 9,8 & $\pm \quad 0,6$ & & \\
\hline & Sur la partie obscure & 7,4 & $\pm \quad 0,8$ & 9,9 & $\pm 0,6$ & & \\
\hline
\end{tabular}

(a) : Moyenne de 11 animaux (7 à tendance diurne, 4 à tendance nocturne) ; (b) : moyenne de 2 animaux (tendance diurne) ; (c) : moyenne de 4 animaux ( 3 à tendance diurne, 1 à tendance nocturne). 
observée. Le profil alimentaire des animaux témoins n'a pas été affecté significativement après la lésion fictive. La réponse des animaux à la lésion ne nous permet pas de signaler de différence en relation avec le sexe.

Animaux à tendance diurne

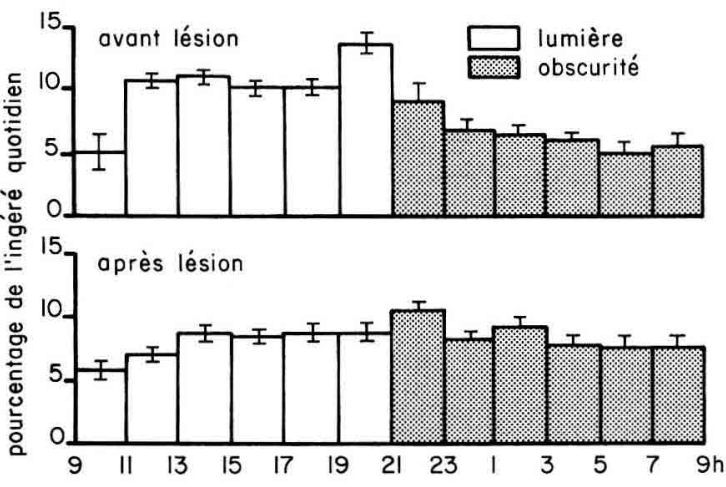

Animaux ò tendance nocturne
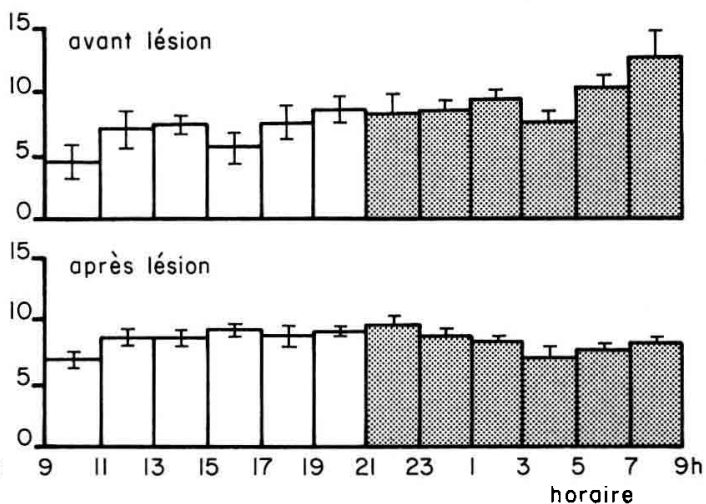

FIG. 1. - Evolution de l'ingéré au cours du nycthémère avant et après lésion des noyaux VMH. (Les écarts-types de la moyenne sont représentés).

TABLEAU 3

Evolution du nombre de repas quotidiens après la lésion des VMH suivant 4 durées différentes du temps de pause maximum au sein d'un repas

\begin{tabular}{|c|c|c|c|c|}
\hline \multirow{2}{*}{ Numéro Oie } & \multirow{2}{*}{$\begin{array}{c}\text { Durée } \\
\text { de l'intervalle } \\
\text { entre } 2 \text { repas }\end{array}$} & Animal Normal & \multicolumn{2}{|c|}{ Animal lésé VMH } \\
\hline & & \multicolumn{2}{|c|}{ Nombre de repas quotidiens } & $\begin{array}{c}\text { Accroissement } \\
\text { (p. 100) }\end{array}$ \\
\hline A & $\begin{array}{l}7 \mathrm{~min} .30 \mathrm{~s} \\
15 \mathrm{~min} . \\
20 \mathrm{~min} . \\
30 \mathrm{~min} . \\
45 \mathrm{~min} .\end{array}$ & $\begin{array}{r}20,4 \pm 0,9 \\
16,5 \pm 0,8 \\
14,5 \pm 0,7 \\
12,3 \pm 0,7 \\
8,8 \pm 0,5\end{array}$ & $\begin{array}{r}31,7 \pm 1,9 \\
26,2 \pm 1,8 \\
22,7 \pm 1,7 \\
16,5 \pm 1,3 \\
8,9 \pm 0,6\end{array}$ & $\begin{array}{l}+55 \\
+59 \\
+56 \\
+\quad 34 \\
+\quad 1\end{array}$ \\
\hline$B$ & $\begin{array}{l}7 \text { min. } 30 \mathrm{~s} \\
15 \mathrm{~min} . \\
20 \mathrm{~min} . \\
30 \mathrm{~min} . \\
45 \mathrm{~min} \text {. }\end{array}$ & $\begin{array}{r}12,4 \pm 1,0 \\
12,0 \pm 0,9 \\
11,7 \pm 0,8 \\
10,6 \pm 0,7 \\
9,8 \pm 0,6\end{array}$ & $\begin{array}{l}20,0 \pm 0,9 \\
18,8 \pm 0,7 \\
17,9 \pm 0,6 \\
16,2 \pm 0,4 \\
12,8 \pm 0,4\end{array}$ & $\begin{array}{l}+61 \\
+57 \\
+53 \\
+53 \\
+31\end{array}$ \\
\hline C & $\begin{array}{l}7 \text { min. } 30 \mathrm{~s} \\
15 \mathrm{~min} . \\
20 \mathrm{~min} . \\
30 \mathrm{~min} . \\
45 \mathrm{~min} .\end{array}$ & $\begin{array}{r}19,8 \pm 1,5 \\
17,6 \pm 1,2 \\
17,1 \pm 1,1 \\
15,1 \pm 0,8 \\
9,0 \pm 0,9\end{array}$ & $\begin{array}{r}36,0 \pm 3,2 \\
28,1 \pm 2,1 \\
26,0 \pm 2,0 \\
17,1 \pm 1,3 \\
9,1 \pm 1,2\end{array}$ & $\begin{array}{l}+82 \\
+60 \\
+52 \\
+13 \\
+\quad 1\end{array}$ \\
\hline $\mathrm{D}$ & $\begin{array}{l}7 \mathrm{~min} .30 \mathrm{~s} \\
15 \mathrm{~min} . \\
20 \mathrm{~min} . \\
30 \mathrm{~min} . \\
45 \mathrm{~min} .\end{array}$ & $\begin{array}{l}17,8 \pm 1,4 \\
16,2 \pm 1,1 \\
15,1 \pm 1,0 \\
14,1 \pm 0,6 \\
10,9 \pm 0,7\end{array}$ & $\begin{array}{l}31,0 \pm 2,2 \\
26,8 \pm 1,6 \\
23,2 \pm 1,2 \\
17,8 \pm 1,2 \\
11,0 \pm 0,6\end{array}$ & $\begin{array}{l}+74 \\
+65 \\
+54 \\
+26 \\
+\quad 0,9\end{array}$ \\
\hline
\end{tabular}

Deux prises alimentaires espacées au maximum de $7 \mathrm{~min} .30 \mathrm{~s}, 15 \mathrm{~min}$, $20 \mathrm{~min}$., $30 \mathrm{~min}$., ont été considérées comme un repas unique. Le tableau rapporte le nombre de repas suivant ces 5 valeurs. 
Nous notons, après le traitement, la persistance d'une corrélation positive entre : - la quantité ingérée et le nombre de repas quotidiens : $r=0,55^{* *}$ $P<0,01 ;-$ la quantité ingérée et la durée quotidienne de prise alimentaire : $\mathbf{r}=0,42^{* *} ;-$ le nombre de repas et la durée quotidienne de prise alimentaire : $r=0,43 * *$.

Afin de vérifier si la définition que nous avons choisie pour le repas pouvait influencer les résultats, nous avons réanalysé la séquence alimentaire des 4 Oies qui présentaient la plus forte augmentation du nombre de repas quotidiens après la lésion. Nous avons observé l'évolution de ce paramètre suivant quatre définitions du critère repas, chez chacun des animaux. Nous présentons dans le tableau 3 l'évolution du nombre de repas quotidiens en fonction des diverses définitions du temps de pause maximum au sein du repas.

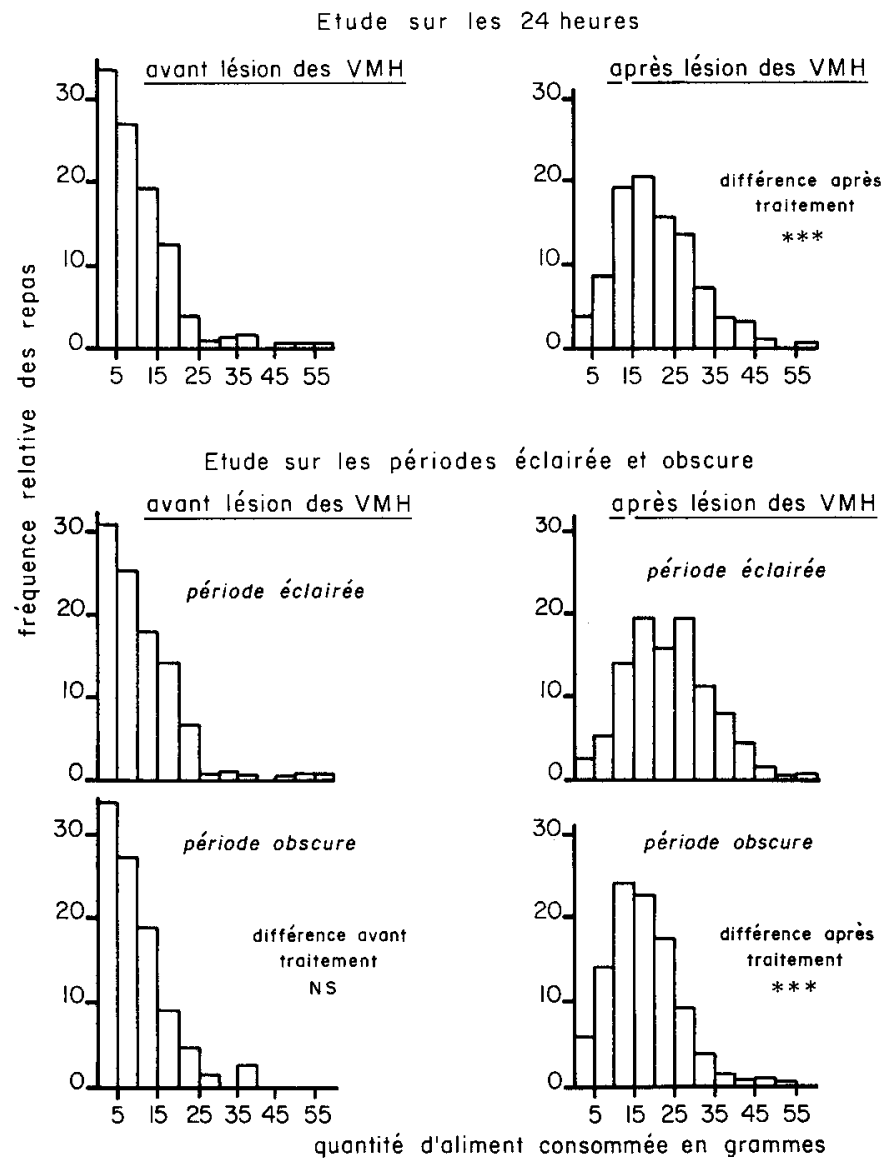

FIG. 2. - Histogrammes des fréquences des quantités d'aliment ingéré par repas. Les histogrammes représentent les différentes valeurs des quantités d'aliment ingéré par repas qui se sont déroulés au cours de 13 nycthémères avant et apres la lesion des noyaux $\mathrm{VMH}$. Les observations portent sur 1026 repas effectués par 2 animaux mâles à tendance diurne (460 repas avant lésion et 556 après lésion). ${ }^{* *}: p<0,001 ; N S$ : Non significatif. 
2. Etude de la distribution des fréquences des quantités ingérées par repas. - Après avoir étudié l'évolution de la fréquence des repas après la lésion, nous avons observé l'évolution des valeurs unitaires de chacun des repas. La figure 2 représente les histogrammes de fréquences des quantités ingérées par repas, les observations portent sur 1026 repas effectués par deux animaux mâles à tendance diurne (460 repas avant lésion et 556 après lésion).

Chez l'Oie normale, la distribution est nettement asymétrique, la plus petite des classes a la plus grande fréquence : l'allure est exponentielle comme chez la Poule (Duncan et al., 1970), le mode étant de $2,5 \mathrm{~g}$ et la moyenne de $10,2 \mathrm{~g}$. Chez l'Oie lésée, mode et moyenne sont pratiquement confondus, leurs valeurs respectives étant de 17,5 et 20,6 : I'histogramme se rapproche ainsi d'une distribution normale.

Avant la lésion, 50 p. 100 des repas sont de moins de $5 \mathrm{~g}$ d'aliment alors qu'après celle-ci 50 p. 100 dépassent $15 \mathrm{~g}$.

En comparant les histogrammes des parties éclairée et obscure, on constate que chez l'Oie normale les distributions des fréquences sont identiques en période

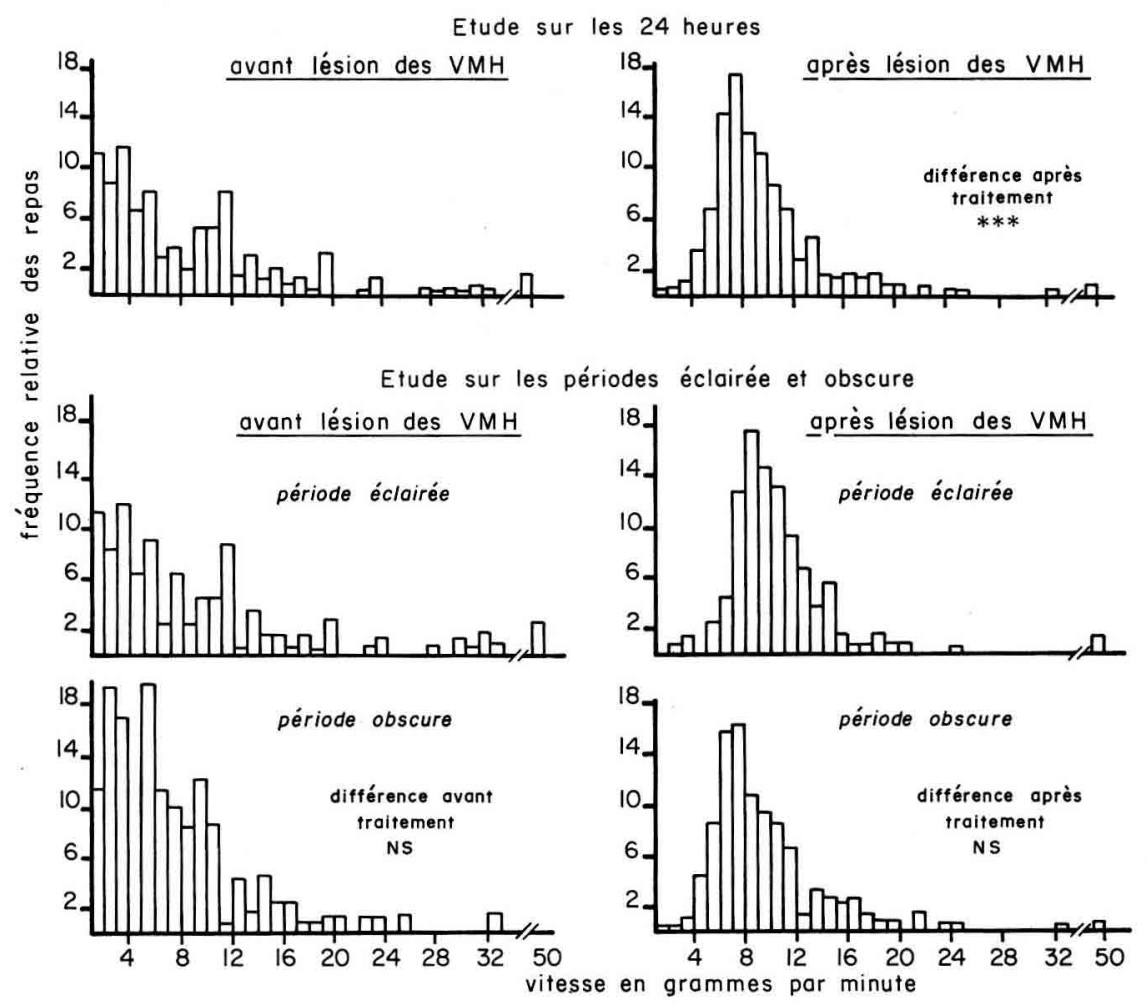

FIG. 3 - Histogrammes des vitesses d'ingestion. Les histogrammes représentent les différentes valeurs des vitesses d'ingestion de l'aliment, enregistrées au cours de 13 nycthémères avant et après lésion des noyaux $\mathrm{VMH}$. Les observations portent sur 1026 repas ingérés par 2 animaux mâles à tendance diurne (460 repas avant lésion et 566 après lésion). ${ }^{* * *}$; $p<0,001$; NS : Non significatif. 
obscure comme en période éclairée, avec cependant une valeur moyenne de l'amplitude du repas légèrement plus faible durant l'éclairement. Chez l'Oie lésée, on note une modification des répartitions des fréquences des quantités ingérées par repas au cours de ces deux parties du nycthémère. C'est en effet en période éclairée que la valeur moyenne du repas a été la plus augmentée et que 50 p. 100 du nombre des repas observés dépassent $20 \mathrm{~g}$, ce même pourcentage dépassant seulement $13 \mathrm{~g}$ en période obscure.

3. Etude des histogrammes de distribution des vitesses d'ingestion. - Nous avons recherché si la lésion des noyaux $\mathrm{VMH}$ modifiait le profil nycthéméral des fréquences des vitesses d'ingestion. C'est ainsi que nous avons construit les histogrammes représentant les fréquences des vitesses d'ingestion observées sur les 1026 repas précédemment cités et reproduits sur la figure 3 .

Ces histogrammes font apparaître la variabilité élevée de la vitesse d'ingestion des repas chez l'animal normal. Les classes de vitesses comprises entre 2 et $12 \mathrm{~g} / \mathrm{min}$. sont toutes bien représentées (80 p. 100 de l'ensemble des repas). Après lésion, les fréquences sont réparties selon une distribution normale. C'est ainsi que les classes se sont resserrées autour du mode $7,5 \mathrm{~g} / \mathrm{min}$. Tout se passe comme si les animaux avaient une vitesse d'ingestion à peu près constante, après la lésion. Par contre, la vitesse moyenne n'a pas varié : elle est de $9,7 \mathrm{~g} / \mathrm{min}$. chez l'animal normal est de $9,9 \mathrm{~g} / \mathrm{min}$. chez l'animal lésé. Seule l'observation des histogrammes fait apparaître le remaniement des distributions : 50 p. 100 des repas s'effectuent à une vitesse dépassant seulement $5 \mathrm{~g} / \mathrm{min}$. chez l'animal avant la lésion, alors qu'après la lésion, la moitié des repas sont ingérés à plus de $8 \mathrm{~g} / \mathrm{min}$. Avant intervention, l'histogramme présente une allure décroissante avec une fréquence plus élevée des repas consommés à faible vitesse, tandis qu'après lésion la plus forte fréquence apparaît dans une classe à valeur plus élevée, les repas consommés à très faible ou à grande vitesse devenant alors plus rares. Les diagrammes des périodes éclairée et obscure ont aussi été modifiés après la lésion quelle que soit la période considérée.

4. Relations entre les durées des intervalles pré- et postprandiaux et la quantité d'aliment consommée au cours du repas. - Nous avons signalé antérieurement au cours d'une étude chez l'Oie normale qu'une grande variabilité de réponse apparaît suivant les animaux. C'est ainsi que pour la moitié des animaux observés la corrélation la plus forte est la relation entre la quantité d'aliment ingérée au cours du repas et la durée de l'intervalle postprandial. Pour l'autre moitié des individus, la relation entre la quantité d'aliment consommée au cours du repas et la durée de l'intervalle préprandial est au contraire la plus élevée. Après la lésion des noyaux $\mathrm{VMH}$, nous notons surtout une corrélation préprandiale qui se manifeste à la fois pendant la période éclairée et la période obscure (tabl. 4).

5. Effet du traitement sur le poids des foies. - Au cours de cette expérience, l'hyperphagie consécutive à la lésion des noyaux ventro-médians de l'hypothalamus a provoqué une augmentation du poids des foies de $238 \mathrm{p} .100$. Les coefficients de variation du poids des foies (écart type $\times 100 /$ movenne) sont 


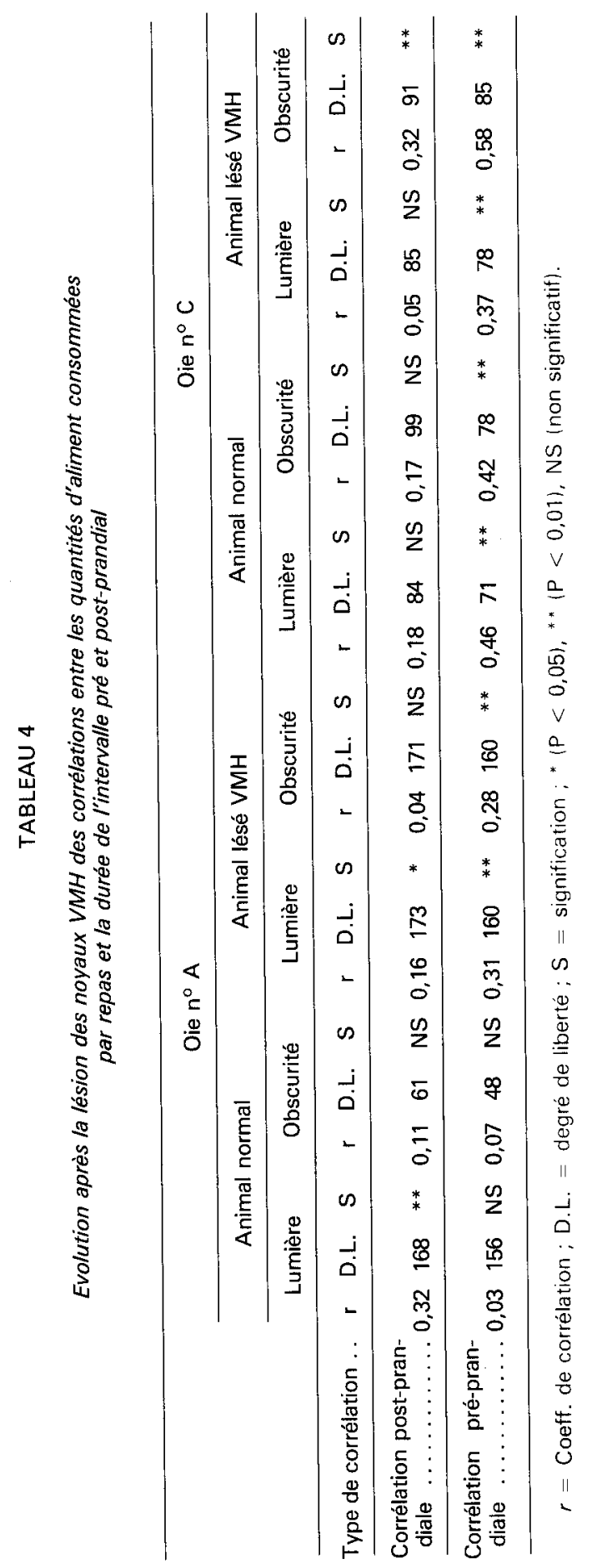


de 23 p. 100 pour les animaux témoins et de 45 p. 100 pour les animaux lésés. Les valeurs moyennes de poids sont les suivantes:

- poids des foies des animaux témoins : $79 \pm 6 \mathrm{~g}$,

- poids des foies des animaux lésés : $267 \pm 33 \mathrm{~g}$.

Cet accroissement pondéral, bien que considérable, reste inférieur à celui obtenu par le gavage traditionnel dans lequel le poids moyen est de $450 \mathrm{~g}$.

\section{Conclusion-Discussion.}

Nous avons souligné dans notre étude l'importance de la zone ventromédiane hypothalamique dans le contrôle de la cyclicité alimentaire. La modification du rythme d'ingestion a déjà été mise en évidence chez le Rat par divers auteurs : Balagura et Devenport (1970), Rietveld et al. (1978), notamment. Chez l'Oie, nous observons une rupture du cycle jour-nuit des consommations avec une nette tendance à l'égalisation de celles-ci au cours des périodes diurne et nocturne. L'effet global de la lésion se manifeste par une hyperphagie qui est la résultante d'une augmentation du nombre de repas, de leur amplitude, de leur durée et de la vitesse à laquelle ils se déroulent. Nous signalons que le profil alimentaire est surtout perturbé dans la partie obscure pour les individus à tendance diurne et dans la partie éclairée pour les individus à tendance nocturne. Ceci pourrait suggérer que la fonction normale des cellules au niveau du noyau VMH lou de structures qui lui sont reliées) serait d'inhiber la prise alimentaire au cours de la période de repos, soit dirune, soit nocturne selon les deux groupes d'Oies identifiés, alors que l'infiuence de ce centre nerveux serait faible au cours de la période alimentaire. La lésion permettrait donc de lever l'inhibition de la prise alimentaire au cours de la période de moindre activité amenant ainsi la rupture du cycle jour-nuit et l'accroissement de la prise alimentaire de façon à la rendre égale pendant les deux phases.

Nous observons chez l'Oie lésée un accroissement quotidien notable du nombre de repas $(+56$ p. 100) ; or chez le Rat ayant subi le même type d'intervention neurochirurgicale il est surtout observé une augmentation de la taille des repas plutôt qu'une augmentation de leur fréquence quotidienne (Larue-Achagiotis et Le Magnen, 1979). II ressort de notre étude (tabl. 3) où nous envisageons diverses définitions du repas que : 1) Quelle que soit la définition choisie l'animal augmente le nombre de ses repas $\left(P<0,001^{* *}\right)$; 2) la différence entre les réponses suivant les divers temps de pause est hautement significative $\left(P<0,001^{* * *}\right)$. L'augmentation du nombre de repas devient relativement moindre lorsque l'on se rapproche de la durée $40 \mathrm{~min}$. (critère retenu pour le Rat).

Si I'on examine le déroulement de la séquence alimentaire de l'Oie lésée on observe que les repas, bien que d'amplitude plus élevée que chez l'animal normal, présentent toujours un début et une fin ; cela est en accord avec ce qui a été observé chez le Rat à savoir que le noyau VMH ne serait pas un « centre de la satiété ". L'hyperphagie serait plutôt l'expression comportementale d'un déséquilibre neuro-endocrinien. Les modifications de l'insulinémie observées chez le Rat porteur de lésions médio-ventrales hypothalamiques (Hales et Kennedy, 1964 ; 
Han et al., 1970 ; Steffens, 1970 ; Louis-Sylvestre, 1976, 1980) confortent cette interprétation. En effet, I'hyperinsulinémie observée par ces auteurs après la lésion des noyaux ventro-médians provoquerait une modification métabolique et par voie de conséquence la rupture de la cyclicité alimentaire. Dans un travail préliminaire (Marcilloux, 1980), où les mesures sont effectuées avec un standard insuline d'Oie, nous observons également une hyperinsulinémie après une telle intervention.

L'Oie présente ainsi divers points de similitude avec le Rat dans la réponse comportementale alimentaire qui fait suite à la lésion des noyaux VMH. Cependant, nous notons chez celle-ci que l'altération du profil alimentaire apparaît dans les deux périodes du nychtémère, ce qui semble être une caractéristique de cette espèce. Toutefois, les perturbations se manifestent surtout au cours de la période qui, chez l'animal normal, correspond à une relative inactivité alimentaire. Une autre particularité peut être notée chez l'Oie par rapport aux mammifères ; nous n'observons pas, en effet, comme il est rapporté chez le Rat, de différence de réponse à la lésion entre mâles et femelles : nous émettons cependant une certaine réserve du fait de l'inégalité dans la répartition des sexes des Oies utilisées. En ce qui concerne les corrélations entre la quantité ingérée au cours du repas et la durée des intervalles pré- ou postprandiaux, nos résultats ne rejoignent pas ceux décrits chez le Rat. Chez cet animal, en effet, ce type de lésion entraîne une corrélation postprandiale dans les deux périodes du nychtémère alors que l'Oie lésée fait apparaître surtout une corrélation préprandiale. Comme nous l'avons montré (Marcilloux et Auffray, 1981) le choix de la définition du repas influence largement les résultats obtenus, nous pensons donc que la différence dans la réponse que nous observons chez l'Oie peut être attribuée à ce facteur de variation.

Nous avons signalé que l'accroissement pondéral des foies gras, bien que très important, demeure insuffisant sur le plan pratique. Ceci peut en partie s'expliquer par la trop courte durée de la phase dynamique. Il a d'ailleurs été montré par Auffray et al. (1973) que si cette période de surconsommation est accrue (suite à l'injection de 6-hydroxydopamine), le poids des foies apparaît sensiblement plus important. Nous pouvons également expliquer la trop faible évolution de la stéatose hépatique par la nature même du régime offert aux animaux. La composition de l'aliment, contrairement à ce qui se pratique lors du gavage traditionnel, ne favorise pas la formation de foie gras car il est équilibré et riche en facteurs lipotropes. II a cependant été mis en évidence par Auffray et Blum (1970) que l'Oie a une certaine affinité pour ce régime équilibré. Cette préférence des animaux hyperphagiques rappelle celle observée chez les mammifères (Agnew et Mayer, 1956). Nous pouvons également émettre l'hypothèse que ce régime distribué ad libitum n'est peut-être pas favorable à l'extension de l'adiposité (MorinJomain, 1966). On peut penser, en effet, que la multiplication du nombre de repas limite le développement de l'obésité et par là même celui du foie. 


\section{Références}

AGNEW L. R. C., MAYER J., 1956. Mechanism of anorexis in vitamin deficient hyperphagic animal. Nature, 177, 1235-1236.

AUfFRAY P., BLUM J. C., 1970. Hyperphagie et stéatose hépatique chez l'Oie après lésion du noyau ventro-médian de I'hypothalamus. C. R. Acad. Sci. Paris, Sér. D, 270, 2362-2365.

AUFFRAY P., MARCILLOUX J. C., BAHY C., ALBE-FESSARD D., 1973. Hyperphagie induite chez I'Oie par injections intraventriculaires de 6-hydroxydopamine. C. R. Acad. Sci. Paris, Sér. $D, 276,347-350$.

BALAGURA S., DEVENPORT L. D., 1970. Feeding patterns of normal and ventromedial hypothalamic lesioned male and female rats. J. comp. physiol. Psychol. 71, 357-364.

CHEVREAU J., BELLOT J., CABANIER M. J., 1977. Formulaires de techniques histologiques, Maloine Ed., p. 227.

DUNCAN I. J. H., HORNE A. R., HUGHES B. O., 1970. The pattern of food intake in female brown Leghorn fowls as recorded in a skinner box. Anim. Behav., 18, 245-255.

HALES C. N., KENNEDY G. C., 1964. Plasma glucose nonesterified fatty acid and insulin concentrations in hypothalamic hyperphagic rats. Biochem. J., 90, 620-624.

HAN P. W., YU Y. K., CHOW S. L., 1970. Enlarged pancreatic itlets of tube fed hypophysectomized rats bearing hypothalamic lesions. Am. J. Physiol., 218, 769-771.

HAWKES M. P. G., GEORGE J. C., 1975. Effects of hypothalamic lesions on levels of plasma free fatty acids in the Mallard duck. Arch. int. Physiol. Bioch., 83, 763-770.

KUENZEL W. J., HELMS C. W., 1967. Obesity produced in a migratory bird by hypothalamic lesions. Biol. Sci., 4, 395-396.

KUPPERMAN M., 1959. A rapid significance test for contingency tables. Biometrics, 15, 625-628.

LARUE-ACHAGIOTIS C., LE MAGNEN J., 1979. The different effects of continuous night at daytime insulin infusion on the meal pattern on normal rats : comparison with the meal pattern of hyperphagic hypothalamic rats. Physiol. Behav., 22, 435-439.

LOUIS-SYLVESTRE J., 1976. Preabsorptive insulin release and hypoglycemia in rats. Amer. J. Physiol., 230, 56-60.

LOUIS-SYLVESTRE J., 1980. Neuroendocrinology of hyperphagias and obesities. Reprod. Nutr. Dévelop., 20, 1545-1562.

LEPKOVSKY S., YASUDA M., 1966. Hypothalamic lesions, growth and body composition of male chickens. Poultry Sci., 45, 582-588.

LEPKOVSKY S., FELDMAN S. E., SHARON I. M., 1967. Food and water intake of the fowl, 117-128. In C. F. CODE, Handbook of physiology, sect. 6, vol. I, Food and Water Intake. Ame. Physiol. Soc., Washington D.C., 117-128.

MARCILLOUX J. C., 1980. Comportement alimentaire de l'Oie de race landaise: importance du noyau ventro-médian de l'hypothalamus dans sa régulation. Effets de la lésion du noyau ventro-médian de l'hypothalamus sur l'insulinémie de l'Oie de race landaise. Th. Doct. Univ., Univ. P. et M. Curie.

MARCILLOUX J. C., AUFFRAY P., 1981. Détermination du profil alimentaire de l'Oie de race landaise. Reprod. Nutr. Dévelop., 21, 737-748.

MORIN-JOMAIN M., 1966. Alimentation discontinue : influence des rythmes alimentaires sur le comportement nutritionnel. Proceed. 7th int. Congr. Nutr., Hamburg, vol. 2, Regulation of hunger and satiety, 224-229.

RIETVELD W. J., TEN HOOR F., KOOIJ M., FLORY W., 1978. Changes in 24-hour fluctuations of feeding behavior during hypothalamic hyperphagia in rats. Physiol. Behav., 21, 615-622.

SNAPIR N., RAVONA, PEREK M., 1973. Effect of electrolytic lesions in various regions of the basal hypothalamus in white Leghorn cockerels upon food intake, obesity, blood plasma triglycerides and protein. Poultry Sci., 52, 629-636.

SNAPIR N., YAAKOBI M., ROBINSON B., 1976. Involvement of the medial hypothalamus and the septal area in the control of food intake and body weight in geese. Pharmacol. biochem. Behav., 5, 609-615.

STEFFENS A. B., 1970. Plasma insulin content in relation to blood glucose level and meal pattern in the normal and hypothalamic hyperphagie rat. Physiol. Behav., 5, 147-151. 